\title{
Sind schwache Leistung und auffälliges Verhalten immer ein Risiko für gelingende Partizipation? Zur Rolle von Kontext und Klassenkohäsion
}

\author{
Sina Schürer $(\mathbb{D}) \cdot$ Stefanie van Ophuysen $(\mathbb{D}) \cdot$ Sophie Marticke $(\mathbb{D}$
}

Eingegangen: 4. November 2020 / Angenommen: 12. März 2021 / Online publiziert: 7. Januar 2022 (C) Der/die Autor(en) 2022

Zusammenfassung Partizipation - speziell hohe Akzeptanz/geringe Ablehnung aller Kinder ist Ziel inklusiver Bildung. Schwache Schulleistungen und Verhaltensprobleme stellen dabei einen Risikofaktor dar, allerdings mit deutlichen Unterschieden zwischen Schulklassen. Inwiefern können diese Unterschiede durch Klassenkohäsion erklärt werden? Gibt es differenzielle Zusammenhänge je nach Partizipationssituation sowie Art des Unterstützungsbedarfs? Für Daten einer standardisierten Befragung von 995 Grundschulkindern aus 45 Klassen wurden hierarchische Regressionsmodelle angepasst. Verhaltensauffälligkeiten und Lernschwierigkeiten sind prädiktiv für geringe Akzeptanz, nur ersteres geht auch mit hoher Ablehnung einher. Kohäsion erhöht Partizipation insgesamt, reduziert jedoch nicht den Nachteil für lernschwache oder verhaltensauffällige Kinder.

Schlüsselwörter Partizipation · Kohäsion · Förderbedarf · Akzeptanz/Ablehnung · Soziometrie · Grundschule

Dr. Sina Schürer $(\bowtie) \cdot$ Prof. Dr. Stefanie van Ophuysen · Sophie Marticke, M.A. Westfälische Wilhelms-Universität, Georgskommende 33, 48143 Münster, Deutschland E-Mail: schuerer@uni-muenster.de

Prof. Dr. Stefanie van Ophuysen

E-Mail: vanophuysen@uni-muenster.de

Sophie Marticke, M.A.

E-Mail: sophie.marticke@uni-muenster.de 


\title{
Are low achievement and behavior problems always a risk for successful participation? The role of context and class cohesion
}

\begin{abstract}
Social participation-especially high acceptance/low rejection-of all children is a goal of inclusive education. Low school performance and behavioural problems are a risk factor here, but with substantial differences between school classes. To what extent can class cohesion explain these differences? Are there differential correlations depending on the participation context as well as the type of students' support needs? Data were analyzed from a standardized survey of 995 elementary school children from 45 classes using hierarchical regression models. Behavioral problems and learning difficulties are predictive of low acceptance; only the former is also associated with high levels of rejection. Cohesion increases overall participation, but does not reduce the disadvantage for children with learning difficulties or behavioral problems.
\end{abstract}

Keywords Participation - Cohesion - Special educational needs - Acceptance/ rejection $\cdot$ Sociometry $\cdot$ Elementary school

\section{Einleitung}

Seit der Unterzeichnung der UN-Behindertenrechtskonvention rückt die Frage nach den Effekten einer gemeinsamen Beschulung aller Kinder vermehrt in den Fokus der Bildungsforschung. Zentral ist dabei die Frage, inwiefern es durch den gemeinsamen Unterricht gelingt, die Partizipation aller Kinder unabhängig von individuellen Fähigkeiten oder Unterstützungsbedarfen, ethnischer oder sozialer Herkunft, Religion oder Geschlecht zu ermöglichen.

Gelingende Partizipation in der Schule meint dabei nicht nur das gleichberechtigte Dabeisein und Mitmachen bei schulischen Aktivitäten, sondern impliziert auch die Anerkennung bzw. die Abwesenheit von Ausgrenzung durch die Mitschüler/innen. So verstanden ermöglicht Partizipation insbesondere die Erfüllung des grundlegenden, menschlichen Bedürfnisses nach Zugehörigkeit (need to belong, Baumeister und Leary 1995). In (experimentellen) Studien konnte belegt werden, dass gravierende negative gesundheitliche, emotionale und kognitive Konsequenzen resultieren, wenn dieses Bedürfnis nicht erfüllt wird (z. B. Baumeister et al. 2005; Hawkley und Cacioppo 2010; Williams 2007). Gleichzeitig kann im schulischen Kontext ein positiver Zusammenhang der Befriedigung dieses Bedürfnisses mit schulischem Wohlbefinden und Schulleistung als belegt gelten (z. B. Wentzel et al. 2014, 2016).

Schule bietet grundsätzlich viele Möglichkeiten für den Aufbau von (positiven) Beziehungen zwischen den Kindern. Dabei kann unterschieden werden zwischen kognitiv-instrumentellen Beziehungen, die in erster Linie der Aufgabenerfüllung im Unterricht dienlich sind (Arbeitspartnerschaften) und affektiv-emotionalen Beziehungen, die in außerunterrichtlichen Situationen erlebt werden (Spielpartnerschaften) (Hannover und Zander 2016). Inwiefern aufgabenbezogene und soziale Partizipation an inklusiv unterrichtenden Grundschulen unabhängig von Schülermerkmalen, konkret Lernschwierigkeiten und Verhaltensauffälligkeiten, gelingt, wird im 
Rahmen des vorliegenden Beitrags untersucht. Weiterhin wird analysiert, inwiefern Kompositionsmerkmale sowie die Kohäsion als Merkmal der Klasse mit individueller Partizipation zusammenhängen.

\section{Akzeptanz und Ablehnung als Facetten sozialer Partizipation}

Das Konstrukt der Partizipation ist facettenreich. Koster et al. (2009) haben in einem Literaturreview verschiedene Definitionen und Operationalisierungen des Konstrukts analysiert und vier Kriterien herausgearbeitet, die indikativ für gelungene Partizipation sind: (1) die Teilnahme an Interaktionen, (2) die Akzeptanz durch Klassenkamerad/innen, (3) das Vorhandensein von Freundschaften und (4) die entsprechenden Wahrnehmungen des Kindes (z. B. subjektiv wahrgenommene Akzeptanz). Während soziale Partizipation durch die Erfassung der Teilnahme an Interaktionen zunächst wertneutral „quantifiziert“ wird, erweitert die Bewertung der Kinder als akzeptierte oder abgelehnte Beziehungspartner/innen das Partizipationsverständnis um eine evaluative Komponente.

In der Schule verbringen Kinder ihre Zeit in unterrichtlichen, aber ebenso in auBerunterrichtlichen Kontexten (z. B. Pausenzeiten). Frederickson und Graham (1999) benennen als Ergebnis ihrer Studie, dass Kinder im Alter zwischen acht und zwölf Jahren ,rated their classmates differentially depending on whether a work context or a play context was being considered“ (S. 19). Entsprechend kann ein Kind als akzeptierte/r Spielpartner/in in positive affektive Peerbeziehungen (social) eingebunden sein. Dies bedeutet jedoch nicht zwangsläufig, dass dieses Kind auch als Arbeitspartner/in akzeptiert wird, da diese Einbindung in kognitiv-instrumentelle Beziehungen (task) stärker von den Fähigkeiten und Leistungen eines Kindes abhängt. Eine gewisse Unabhängigkeit hinsichtlich der Partizipation in diesen beiden Kontexten erscheint somit plausibel.

Diese Vorüberlegungen lassen vermuten, dass Kinder mit schwachen Schulleistungen andere Schwierigkeiten bei der Partizipation erleben als Kinder mit auffälligen, insbesondere externalisierenden, Verhaltensweisen. So könnten Kinder mit Lernschwächen nur geringe Akzeptanz als Arbeitspartner/in erfahren, ohne dass dies Auswirkungen auf ihre außerschulische, soziale Akzeptanz haben muss. Hingegen stellen Kinder mit problematischem Verhalten ihre Mitschüler/innen sowohl in kognitiv-instrumentellen als auch in affektiv-sozialen Situationen vor besondere Herausforderungen, sodass anzunehmen ist, dass sich sowohl ihre aufgabenbezogene als auch ihre soziale Partizipation eher schwierig gestaltet. Eine differenzierte Analyse von Akzeptanz bzw. Ablehnung, bei der sowohl der Partizipationskontext (task bzw. social) als auch die Art des Unterstützungsbedarfs (Lernen bzw. Verhalten) berücksichtigt werden, erscheint somit notwendig.

\section{Prädiktoren von Akzeptanz/Ablehnung}

Studien zur Partizipation in Schulklassen nehmen in der Regel Kinder mit besonderem Förderbedarf - insbesondere in den Förderschwerpunkten (FSP) emotional- 
soziale Entwicklung (ESE) oder Lernen (LE) - in den Blick. Bei der Interpretation dieser Studien ist zu beachten, dass der Begriff des ,,besonderen Förderbedarfs“ (special educational need) oft informell verwendet wird, obwohl also gar keine formale Diagnose zum sonderpädagogischen Förderbedarf (SPF) zugrunde liegt. Vielmehr werden häufig (kategoriale) Einschätzungen der Lehrkräfte (z. B. Huber und Wilbert 2012: dreistufige Skala (SPF gering, mittel, hoch), Lehrkrafturteile zur Schulleistung (5-stufig); Krull et al. 2018: Vermutung der Lehrkraft über den SPF (vorhanden vs. nicht vorhanden)) oder Ergebnisse von standardisierten Leistungstests oder Verhaltensbeurteilungen (z.B. Spilles et al. 2019) genutzt.

\subsection{Prädiktoren auf Individualebene}

In Studien, die nicht nach FSP differenzieren, erweisen sich Kinder mit SPF im Vergleich zu ihren Mitschüler/innen als weniger akzeptiert und häufiger abgelehnt (z.B. Avramidis 2013; Cambra und Silvestre 2003; Grütter et al. 2015; Nepi et al. 2013, 2015; Pijl et al. 2008). Sie sind zudem häufiger in Risiko-Statusgruppen zu finden (z.B. Pijl et al. 2008).

Speziell für Kinder mit Lernschwierigkeiten liegen mittlerweile umfangreiche Forschungsergebnisse vor. Die international vergleichende Metaanalyse von Kavale und Forness (1996) findet über 152 Studien hinweg einen starken Zusammenhang zwischen Lernbeeinträchtigung und sozialer Ablehnung bei Schüler/innen aller Altersstufen. Dieser Befund wird in aktuelleren Überblicksarbeiten bestätigt (Bless 2000; Bless und Mohr 2007; Nowicki 2003). Für Kinder mit SPF im Bereich ESE ist der Forschungsstand hingegen schmaler, belegt jedoch auch eine stärkere Ablehnung von Kindern mit Verhaltensauffälligkeiten im Vergleich zu ihren Mitschüler/ innen ohne entsprechenden Förderbedarf (Avramidis 2013; Monchy et al. 2004).

In einem Überblick über Studien seit dem Jahr 2010, die die Partizipation von Grundschulkindern mit vs. ohne SPF in Deutschland analysieren, findet Schürer (2020) nur zehn Veröffentlichungen, die sich mit der Akzeptanz bzw. Ablehnung beschäftigen. Insgesamt stimmen alle Befunde darin überein, dass Kinder mit SPF weniger akzeptiert und häufiger abgelehnt werden als ihre Mitschüler/innen ohne SPF. Nur eine dieser Studien (Krull et al. 2018) vergleicht die Partizipation von Kindern mit unterschiedlichen FSPen. Die Autor/innen zeigen, dass die Ablehnung für Kinder mit SPF im Bereich LE geringer ausfällt als für Kinder mit Beeinträchtigungen im Bereich ESE.

Die Operationalisierung von Akzeptanz und Ablehnung erfolgt in diesen Studien über soziometrische Methoden. So wird erfasst, wie häufig ein Kind von Mitschüler/ innen als gewünschte/r Sitznachbar/in (Huber 2011; Huber und Wilbert 2012; Krull et al. 2018; Kulawiak und Wilbert 2015) oder als Freund/in (Henke et al. 2017) benannt wird. Einige Studien erfassen mehrere Kriterien (z. B. Sitznachbar/in, Arbeitspartner/in), wobei diese anschließend verrechnet werden (Krawinkel et al. 2017). Die von uns vorgeschlagene Unterscheidung zwischen Partizipation in sozialen außerunterrichtlichen Situationen (social) sowie im Bereich des Unterrichts (task), wird bei keiner dieser Studien berücksichtigt.

Auch in internationalen Studien wird nur selten der Blick explizit auf die inhaltlichen Kontexte der Partizipation gelegt. Eine Ausnahme bilden Frederickson 
und Furnham (2001), die den soziometrischen Status über den Social Inclusion Survey (SIS, Frederickson und Graham 1999) erfassen, welcher die beiden Teilbereiche „like to play“ und „like to work“ unterscheidet. Ihre Ergebnisse zeigen, dass Kinder mit Lernschwierigkeiten in beiden Bereichen weniger akzeptiert und häufiger abgelehnt werden. Eine vergleichende Analyse der Akzeptanz von Kindern mit Unterstützungsbedarf im Bereich LE und ESE für diese beiden Kontexte der Partizipation ist bisher jedoch nicht umfassend erfolgt.

Schürer (2019) untersucht mit der subjektiv wahrgenommenen Partizipation und der Anzahl reziproker Beziehungen zwei andere Facetten der Partizipation. Sie differenziert zwischen Kindern mit schwachen Schulleistungen bzw. auffälligem Verhalten und unterscheidet zwischen den beiden Kontexten „Spielen“ vs. „Arbeiten“. Die Befunde bestätigen für den Bereich des Spielens und des Arbeitens in gleicher Weise: Je höher der Grad der Verhaltensauffälligkeit desto geringer die selbstwahrgenommene Partizipation und die Anzahl reziproker Beziehungen. Die Leistung ist hingegen nur prädiktiv für die Partizipation in Arbeitssituationen. Ob sich dies auch für die Akzeptanz bzw. die Ablehnung als weiteren Indikator der Partizipation bestätigen lässt, ist bislang nicht überprüft.

\subsection{Prädiktoren auf Klassenebene}

Empirische Studien weisen darauf hin, dass nennenswerte Unterschiede im Ausmaß der Partizipation zwischen Klassen auftreten (z. B. Huber und Wilbert 2012; Krull et al. 2014). Daher ist es naheliegend, neben Situations- und Personenmerkmalen auch Merkmale auf Klassenebene in den Blick zu nehmen. So könnten Unterschiede in der Partizipation auch durch die spezifische Zusammensetzung der Schulklasse - beispielsweise hinsichtlich des Jungenanteils oder der ethnischen Heterogenität sowie bezogen auf das mittlere Leistungsniveau oder die Anzahl an Kindern mit Verhaltensauffälligkeiten - erklärt werden. Doch während Effekte der Klassenkomposition auf die Leistung als gut belegt gelten können (Dumont et al. 2013; Van Ewijk und Sleegers 2010), wurden die Auswirkungen auf soziale Konsequenzen des Miteinanders bisher nur selten untersucht (Cook und Semmel 1999; Scharenberg et al. 2019).

Neben der Komposition der Schulklasse scheint auch ein positives Klassenklima förderlich für die individuelle Partizipation zu sein. In einer Studie von Krawinkel et al. (2017) zeigen sich signifikante Zusammenhänge zwischen der sozialen Partizipation und dem Klassenklima, wobei insbesondere Kinder mit SPF von einem guten Klassenklima profitieren. Sie schlussfolgern daher: „Das erhöhte Ausgrenzungsrisiko von Kindern mit SPF lässt sich durch ein gutes Klassenklima [...] mindern“ (Krawinkel et al. 2017, S. 291). Das Klassenklima wird über die gleichnamige Subskala des FEESS 3-4 (Rauer und Schuck 2003) erfasst, deren Items eine deutliche Nähe zum Konstrukt der Kohäsion zeigen.

Kohäsion gilt als wichtiges Merkmal sozialer Gruppen und kann verstanden werden als ,the tendency for a group to stick together and remain united in the pursuit of its instrumental objectives and/or for the satisfaction of member affective needs" (Carron et al. 1998, S. 213). In Analogie zu unserer Differenzierung von aufgabenbezogener und sozialer Partizipation lenkt diese Definition die Aufmerksamkeit auf 
die Funktion der Gruppe, nämlich das Erfüllen gemeinsamer Aufgaben (task) sowie die Befriedigung des Bedürfnisses nach Zugehörigkeit (social). In diesem Sinne können Schulklassen gleichermaßen als sozialorientierte als auch als aufgabenorientierte Gruppen betrachtet werden. In einer hoch kohäsiven Schulklasse besteht somit eine starke Verbundenheit zwischen den Kindern der Klasse, die bezogen sein kann auf die gemeinsam zu erfüllenden Aufgaben im Unterricht oder auf soziale, außerunterrichtliche Aktivitäten. Je nach Klasse kann die Ausprägung der sozialen oder aufgabenbezogenen Kohäsion unterschiedlich stark sein (Schürer et al. 2020). In Ergänzung der Befunde zum Klassenklima von Krawinkel et al. (2017) erscheint es daher spannend, die Kohäsion als Klassenmerkmal in seiner Auswirkung auf Akzeptanz/Ablehnung in den Blick zu nehmen.

\section{Forschungsfragen}

Zusammenfassend zeigt sich, dass über verschiedene Länder und Schulsysteme hinweg Kinder mit besonderem Unterstützungsbedarf zu einer Risikogruppe in Bezug auf ihre Akzeptanz und Ablehnung gehören. Kritisch muss jedoch berücksichtigt werden:

1. Differenzierung verschiedener Partizipationskontexte: In der Schule sind zwei zentrale Inhaltsbereiche zu unterscheiden. Partizipation kann im Rahmen des Unterrichts, aber auch in außerunterrichtlichen Situationen erfolgen. Eine Überprüfung der Annahme, ob sich das Ausgrenzungsrisiko je nach Kontext unterscheidet, wurde bisher nicht systematisch vorgenommen.

2. Differenzierung unterschiedlicher Unterstützungsbedarfe: In vielen Studien werden Kinder mit verschiedenen FSPen zusammengefasst. Insbesondere werden die drei FSPe LE, Sprache und ESE oft unter dem Oberbegriff der Entwicklungsstörungen zusammengefasst und gemeinsam betrachtet (z. B. Henke et al. 2017; Huber und Wilbert 2012). Diese Vorgehensweise erscheint problematisch: In den wenigen Studien, die eine getrennte Betrachtung der FSPe vornehmen, stellen vornehmlich Kinder mit dem FSP ESE eine besondere Risikogruppe dar (z. B. Krull et al. 2018; Mand 2007; Zurbriggen und Venetz 2016). Inwiefern sich zusätzliche differenzielle Effekte je nach Partizipationskontext (social vs. task) ergeben, ist eine weitere ungeklärte Frage.

3. Berücksichtigung von Klassenmerkmalen: In wenigen Studien wird das Augenmerk auf Kompositionsmerkmale oder Kontextfaktoren als Prädiktoren von Akzeptanz/Ablehnung gerichtet. Ein solcher Kontextfaktor ist die Klassenkohäsion. Entsprechende Analysen stehen noch aus.

Im Rahmen dieser Studie überprüfen wir daher, inwiefern sich die Akzeptanz/ Ablehnung von Grundschulkindern in schulischen Lern- und Arbeitsprozessen (task) sowie in außerunterrichtlichen Aktivitäten (social) auf Individualebene durch ihre Leistung(sschwäche) und Verhaltensauffälligkeiten sowie auf Gruppenebene durch Kompositionsmerkmale und die Kohäsion der Klasse vorhersagen lässt. Konkret erwarten wir: 
I. Schwache Leistung bzw. starke Verhaltensauffälligkeit gehen mit geringer Akzeptanz bzw. starker Ablehnung einher.

II. Der Effekt der Leistung zeigt sich nur für die aufgabenbezogene Partizipation. Verhaltensauffälligkeit ist hingegen sowohl für die aufgabenbezogene als auch die soziale Partizipation prädiktiv.

III. Die individuelle Akzeptanz/Ablehnung hängt auch von Merkmalen der Klassenkomposition ab.

IV. Je kohäsiver eine Klasse ist, desto höher fallen die Werte für Akzeptanz und desto niedriger die Werte für Ablehnung aus.

V. In kohäsiven Klassen ist der Zusammenhang von Leistung und Verhaltensauffälligkeit mit den Partizipationskennwerten geringer als in Klassen mit geringer Kohäsion.

\section{Methode}

\subsection{Stichprobe}

Die Daten stammen aus dem SoPaKo-Projekt (Soziale Partizipation durch Kohäsion), einer Interventionsstudie an Grundschulen in Nordrhein-Westfalen. In die Analysen gehen Daten von 1013 Zweit- und Drittklässler/innen aus 45 Grundschulklassen ein (Jg. 2: 478, Jg. 3: 535 Kinder). 51,7\% sind weiblich und 47,7\% haben einen Migrationshintergrund (gesprochene Sprache zu Hause „Nicht Deutsch“ oder „Deutsch und eine andere Sprache“). Insgesamt handelt es sich um eine vergleichsweise leistungsschwache Gruppe von Kindern (siehe Tab. 1). In einem Mathematikleistungstest (DEMAT 1+ bzw. 2+, Krajewski et al. 2002a, b) erreichen die Kinder im Mittel einen Prozentrang von 35,4, im Lesestest (ELFE; Lenhard et al. 2017) liegt der mittlere Prozentrang bei 39,8. Dies ist nicht unerwartet mit Blick darauf, dass viele Schulen der Stichprobe dem VERA-Standorttyp ${ }^{1}$ Stufe 4 oder 5 angehören. Hinsichtlich der Verhaltensauffälligkeit ist die Stichprobe als durchschnittlich zu bezeichnen. 81,1\% der Kinder können aufgrund der Lehrkrafteinschätzung mittels des Strengths and Difficulties Questionnaire (SDQ, Goodman 1997) als unauffällig, $8,0 \%$ als grenzwertig und 10,9\% als auffällig in ihrem Problemverhalten kategorisiert werden.

Tab. 1 Deskriptive Kennwerte der Leistungstests zur Beschreibung der Stichprobe

\begin{tabular}{llllll}
\hline & M (SD) & Min/Max & 25. Perzentil & 50. Perzentil & 75. Perzentil \\
\hline PR DEMAT & $35,4(27,9)$ & $0 / 99$ & 13,6 & 34,5 & 61,8 \\
PR ELFE & $39,8(28,6)$ & $0,8 / 98,8$ & 11 & 28 & 58 \\
\hline
\end{tabular}

PR Prozentrang

\footnotetext{
1 Der VERA-Standorttyp ermöglicht einen fairen Vergleich der Ergebnisse der Lernstandserhebungen. Schulen werden entsprechend ihrer Rahmenbedingungen, z. B. bzgl. der Zusammensetzung der Schülerschaft sowie der regionalen Struktur im Umfeld der Schule, einem Standorttypen zugeordnet. Die Schülerschaft von Schulen des Standorttyps 4 bzw. 5 weist dabei einen vergleichsweisen hohen Anteil an Kindern mit Migrationshintergrund auf und stammt aus sozialschwachen Familien.
} 


\subsection{Instrumente \& Maße}

\subsubsection{Abhängige Variablen}

Zur Erfassung der sozialen und aufgabenbezogenen Akzeptanz/Ablehnung beurteilte jedes Kind auf einem fünfstufigen Antwortformat alle anderen Kinder seiner Klasse hinsichtlich der Fragen „Wie gut kannst du mit XY arbeiten?“ (task) und „Wie gerne spielst du mit XY?“ (social). Die an der Klassengröße relativierte Anzahl der extrem positiven Ratings, die ein Kind von seinen Mitschüler/innen erhalten hat, wurde als Akzeptanzwert genutzt. Der Ablehnungswert wurde analog über die extrem negativen Ratings gebildet.

\subsubsection{Prädiktoren auf Individualebene}

Anstelle einer kategorialen Zuordnung (mit vs. ohne Förderbedarf LE/ESE) wurde für jedes Kind die Leistung über die oben genannten standardisierten Leistungstests erhoben. Die Testwerte wurden z-standardisiert und zu einem Kennwert für die Leistung zusammengefasst.

Auch die Erfassung der Verhaltensauffälligkeiten erfolgte als metrische Variable mithilfe des SDQ (Goodman 1997) in der Lehrkräfteversion. Die Werte der vier „Problem“-Skalen (Emotionale Probleme, Verhaltensprobleme, Hyperaktivität, Probleme mit Gleichaltrigen) wurden zu einem Gesamtwert aufsummiert und z-standardisiert.

\subsubsection{Prädiktoren auf Klassenebene}

Merkmale der Klassenkomposition wurden durch Aggregierung von Individualdaten ermittelt. So wurden der Anteil an Mädchen, der Anteil an Kindern mit Migrationshintergrund, die mittlere Leistung und der mittlere SDQ als Klassenmerkmale berechnet.

Die Klassenkohäsion wurde analog zur Akzeptanz/Ablehnung getrennt für die Kontexte Lernen und Arbeiten (task) sowie außerunterrichtliche Aktivitäten (social) erhoben. Es wurde je ein sozio- und ein psychometrisches Maß genutzt. Auf Basis soziometrischer Nominationsdaten (,Mit welchen Kindern spielst du am meisten?“, „Mit welchen Kindern kannst du am besten arbeiten?“) wurde pro Kontext die Dichte berechnet; d.h. die Anzahl der jeweils realisierten Nominationen in einer Klasse wurde dividiert durch die Zahl der möglichen Nominationen (z.B. Jansen 2006). Die Dichte beschreibt als einer der geläufigsten soziometrischen Kennwerte,

Tab. 2 Skalen des GruKo4

\begin{tabular}{lllll}
\hline Skala & Beispielitem & Itemanzahl & Cronbachs $\alpha$ & M (SD) \\
\hline GI_social & $\begin{array}{l}\text { In meiner Klasse halten wir alle gut zu- } \\
\text { sammen }\end{array}$ & 3 & 0,768 & $3,33(0,75)$ \\
GI_task & $\begin{array}{l}\text { Meine Klasse ist eine richtig gute Lernge- } \\
\text { meinschaft }\end{array}$ & 4 & 0,733 & $3,35(0,69)$ \\
\hline
\end{tabular}

GI Group Integration 
wie stark die Schüler/innen ihrer Klasse vernetzt sind (z. B. Borgatti et al. 2018; Prell 2012). Zusätzlich schätzte jedes Kind den Gruppenzusammenhalt der Klasse auf zwei psychometrischen Skalen des GruKo4 ein (Schürer et al. 2020). Die Skalen bestehen aus vier bzw. drei Items mit einem vierstufigen Antwortformat (,,stimmt gar nicht“ bis „stimmt genau“). Beispielitems sowie Angaben zur internen Konsistenz sind Tab. 2 zu entnehmen.

\subsection{Durchführung}

Die Daten für die hier vorgestellten Analysen stammen aus der ersten Erhebung der Interventionsstudie, die zu Beginn des Schuljahres 2017/18 stattfand (mit Ausnahme der Daten des ELFE II, die zum zweiten Messzeitpunkt in der Mitte des Schuljahres erhoben wurden). Die Befragung der Schüler/innen wurde von geschulten Testleiter/ innen administriert. Standardisierte Testleitungsmanuale gewährleisteten einen einheitlichen Ablauf. Die Schulleistungstests wurden im Klassenverband jeweils in der ersten Schulstunde durchgeführt. Im Anschluss bearbeiteten die Kinder einen Fragebogen und die soziometrischen Erhebungsbögen. Dies erfolgte in Jahrgang drei im Klassenverband, in Jahrgang zwei hingegen in Kleingruppen.

\subsection{Auswertungsmethoden}

Die Zusammenhangsannahmen wurden unter Berücksichtigung der Zwei-EbenenStruktur der Daten mittels hierarchischer linearer Modelle mit dem Programm HLM 6.08 (Raudenbush et al. 2009) überprüft. Hierarchische lineare Modelle können zunächst überprüfen, wie sich die Gesamtvarianz auf die zwei Analyseebenen - Unterschiede zwischen den Schulklassen (Level 2) bzw. Unterschiede zwischen den Individuen (Level 1) - aufteilt. Unterschiede zwischen den Schulklassen können sich dabei einerseits auf den Intercept (in unserem Fall also die durchschnittliche Partizipation der Kinder einer Klasse) oder auf die Steigungsparameter beziehen. Letzteres läge beispielsweise vor, wenn die Abhängigkeit der Partizipation von der individuellen Leistung in den Klassen unterschiedlich stark ausfallen würde. In einem weiteren Schritt kann überprüft werden, inwiefern die Varianzanteile, durch die gewählten Prädiktoren auf den beiden Ebenen reduziert werden können. Indem Steigungsparameter als zwischen den Klassen variierend modelliert werden, können auch sogenannte Cross-Level-Interaktionen überprüft werden. Auf diese Weise kann beispielsweise analysiert werden, ob in kohäsiven Klassen der Zusammenhang zwischen Leistung und Partizipation geringer ausfällt als in Klassen mit einer geringen Gruppenkohäsion.

Für jede Partizipationsvariable wurde eine separate Analyse durchgeführt. Zunächst wurde jeweils ein Nullmodell ganz ohne Prädiktoren angepasst, um die Intraklassenkorrelation (ICC) und damit die Varianzanteile auf Individual- und Gruppenebene zu bestimmen. Im zweiten Schritt wurden die beiden dummy-kodierten Variablen Geschlecht $(0=$ weiblich, $1=$ männlich) und Migrationshintergrund $(0=$ deutsche Familiensprache, $1=$ nicht deutsche Familiensprache $)$ sowie der Leistungs- und der SDQ-Wert (beide am Gruppenmittelwert zentriert) als Prädiktoren auf Individualebene in das Modell aufgenommen. Mit diesem Analyseschritt wurde 
nicht nur der Zusammenhang der Individualmerkmale mit der Partizipation überprüft, sondern auch, ob die Stärke des Zusammenhangs der Partizipation mit den Individualmerkmalen zwischen den Klassen variiert. Wenn dies der Fall war, wurde im finalen Modell ein zufälliger Steigungsparameter (random slope) genutzt, andernfalls wurde der Steigungsparameter als nicht zufällig (fixed slope) modelliert. In dem resultierenden „Intercept-and-Slopes-as-Random“-Modell wurden sodann Prädiktoren auf der Gruppenebene aufgenommen, um deren Effekt auf den Intercept und die zufälligen Steigungsparameter zu überprüfen. Auf Gruppenebene gingen die oben genannten aggregierten Individualmerkmale ein, um eventuelle Kompositionseffekte abzubilden. Ebenfalls wurden die Kohäsionsvariablen als Prädiktoren auf Gruppenebene eingesetzt. Zur Vorhersage der sozialen Partizipation wurden jeweils die am Gesamtmittelwert zentrierten Variablen Dichte_social und GI_social genutzt und zur Vorhersage der aufgabenbezogenen Partizipation die Variablen Dichte_task und GI_task.

\section{Ergebnisse}

\subsection{Deskriptive Befunde}

In Tab. 3 sind die Mittelwerte der erhaltenen Extrembewertungen dargestellt. Durchschnittlich erhält ein Kind zwischen fünf und knapp sieben extreme Bewertungen. Die durchschnittlichen extremen Ablehnungswerte als Spielpartner/in $(6,98)$ fallen dabei höher aus als die extremen Ablehnungswerte als Arbeitspartner/in $(M=5,51)$. Die Korrelationen zwischen den Variablen fallen moderat bis hoch aus. Insbesondere die Korrelationen zwischen den jeweiligen beiden task- und social-Variablen verweisen auf deutliche Zusammenhänge (Akzeptanz: $r=0,766$; Ablehnung: $r=0,858$ ).

\subsection{Hierarchische lineare Modellierung}

Die ICCs der Akzeptanzwerte liegen bei 0,173 (task) und 0,185 (social) sowie bei 0,322 (task) und 0,365 (social) für die Ablehnungswerte. Je nach Indikator der Partizipation liegen somit zwischen $17 \%$ und knapp $37 \%$ der Varianz auf Gruppenebene, sodass die Berücksichtigung der Gruppenebene bzw. der Level 2 Variablen angezeigt erscheint.

Tab. 3 Deskriptive Kennwerte der abhängigen Variablen: Mittelwerte $(M)$, Standardabweichungen $(S D)$, Korrelationen

\begin{tabular}{lllll}
\hline & $\mathrm{M}(\mathrm{SD})$ & $(1)$ & $(2)$ & $(3)$ \\
\hline (1) Akzeptanz_social & $6,12(3,48)$ & - & - & - \\
(2) Akzeptanz_task & $6,72(3,84)$ & $0,766^{* *}$ & - & - \\
(3) Ablehnung_social & $6,98(4,47)$ & $-0,428^{* *}$ & $-0,433^{* *}$ & - \\
(4) Ablehnung_task & $5,51(4,28)$ & $-0,434^{* *}$ & $-0,500^{* *}$ & $0,858^{* *}$ \\
\hline
\end{tabular}

$N_{\text {Kinder }}=995, N_{\text {Klassen }}=45$

$* * p<0,001$ 
Tab. 4 Varianz der zufälligen Effekte auf Level 1 und Level 2 (Intercept und Steigungsparameter)

\begin{tabular}{lllllllll}
\hline & \multicolumn{2}{c}{ Akzeptanz Social } & \multicolumn{2}{c}{ Akzeptanz Task } & \multicolumn{2}{c}{ Ablehnung Social } & \multicolumn{2}{c}{ Ablehnung Task } \\
Zufällige Effekte & Var.kom. & $\chi^{2}$ & Var.kom. & $\chi^{2}$ & Var.kom. & $\chi^{2}$ & Var.kom. & $\chi^{2}$ \\
\hline Level 1 & 0,0123 & - & 0,0116 & - & 0,0137 & - & 0,0115 & - \\
Level 2 & & & & & & & & \\
Intercept & $0,0087^{* * *}$ & 216,9 & $0,0088^{* * *}$ & 233,8 & $0,0141^{* * *}$ & 353,4 & $0,0122^{* * *}$ & 316,5 \\
Slope Migration & $0,0013^{*}$ & 61,9 & 0,0003 & 37,4 & $0,0015^{*}$ & 66,1 & 0,0013 & 53,3 \\
Slope Geschlecht & $0,0033^{* * *}$ & 92,0 & $0,0032^{* * *}$ & 87,5 & $0,0027^{* *}$ & 73,9 & $0,0021^{* *}$ & 77,0 \\
Slope SDQ & $0,0007^{* * *}$ & 72,9 & $0,0006^{* * *}$ & 72,2 & $0,0010^{* * *}$ & 90,3 & $0,0023^{* * *}$ & 145,7 \\
Slope Leistung & 0,0001 & 47,0 & 0,0001 & 45,6 & 0,0003 & 49,8 & $0,0004^{* *}$ & 70,5 \\
\hline
\end{tabular}

df $=44$

$* * * p<0,001, * * p<0,01, * p<0,05$

Tab. 5 Zwei-Ebenen-Regressionsanalysen für Akzeptanz/Ablehnung im außerunterrichtlichen (social) und unterrichtlichen (task) Kontext

\begin{tabular}{|c|c|c|c|c|c|c|c|c|}
\hline \multirow[b]{2}{*}{ Feste Effekte } & \multicolumn{2}{|c|}{ Akzeptanz Social } & \multicolumn{2}{|c|}{ Akzeptanz Task } & \multicolumn{2}{|c|}{ Ablehnung Social } & \multicolumn{2}{|c|}{ Ablehnung Task } \\
\hline & Koeff. & $\mathrm{SE}$ & Koeff. & SE & Koeff. & $\mathrm{SE}$ & Koeff. & $\mathrm{SE}$ \\
\hline \multicolumn{9}{|l|}{ Individualebene } \\
\hline Intercept & $0,302^{* * * *}$ & 0,012 & $0,337^{* * * *}$ & 0,013 & $0,278^{* * *}$ & 0,014 & $0,225^{* * *}$ & 0,013 \\
\hline Migration & $-0,053^{* * * *}$ & 0,009 & $-0,047^{* * * *}$ & 0,007 & $0,063^{* * *}$ & 0,010 & $0,045^{* * *}$ & 0,010 \\
\hline Geschlecht & 0,004 & 0,011 & $-0,012$ & 0,011 & 0,014 & 0,011 & 0,011 & 0,010 \\
\hline SDQ & $-0,073^{* * *}$ & 0,006 & $-0,091^{* * *}$ & 0,005 & $0,085^{* * * *}$ & 0,006 & $0,101^{* * * *}$ & 0,008 \\
\hline Leistung & $0,015^{* *}$ & 0,004 & $0,027^{* * *}$ & 0,004 & $-0,010$ & 0,005 & $-0,011$ & 0,006 \\
\hline \multicolumn{9}{|l|}{ Gruppenebene } \\
\hline Migration_Anteil & $0,063^{*}$ & 0,026 & 0,012 & 0,051 & $-0,134$ & 0,074 & $-0,139^{*}$ & 0,066 \\
\hline Mädchen_Anteil & 0,104 & 0,056 & 0,058 & 0,080 & 0,161 & 0,105 & 0,205 & 0,118 \\
\hline SDQ_MW ${ }^{\mathrm{a}}$ & 0,012 & 0,025 & 0,049 & 0,028 & $-0,046$ & 0,030 & 0,035 & 0,034 \\
\hline Leistung_MW ${ }^{\mathrm{a}}$ & 0,004 & 0,014 & $-0,006$ & 0,022 & $-0,175^{* * *}$ & 0,027 & $-0,139^{* * *}$ & 0,025 \\
\hline Kohäsion_GI ${ }^{b}$ & $0,064^{* *}$ & 0,022 & 0,037 & 0,026 & $-0,128^{* * * *}$ & 0,033 & $-0,093^{*}$ & 0,035 \\
\hline Kohäsion_Dichte ${ }^{b}$ & $0,032^{* * *}$ & 0,004 & $0,027^{*}$ & 0,011 & $-0,015$ & 0,011 & $-0,009$ & 0,013 \\
\hline \multicolumn{9}{|c|}{ Cross-Level-Interaktion } \\
\hline $\begin{array}{l}\text { SDQ × Kohä- } \\
\text { sion_GI }\end{array}$ & $-0,021$ & 0,013 & 0,019 & 0,010 & $-0,024$ & 0,021 & $-0,057^{*}$ & 0,021 \\
\hline $\begin{array}{l}\text { SDQ } \times \text { Kohä- } \\
\text { sion_Dichte }\end{array}$ & $-0,001$ & 0,007 & $-0,005$ & 0,007 & $-0,007$ & 0,005 & $-0,005$ & 0,007 \\
\hline $\begin{array}{l}\text { Leistung } \times \text { Kohä- } \\
\text { sion_GI }\end{array}$ & - & - & - & - & - & - & 0,007 & 0,017 \\
\hline $\begin{array}{l}\text { Leistung } \times \text { Kohä- } \\
\text { sion_Dichte }\end{array}$ & - & - & - & - & - & - & 0,004 & 0,004 \\
\hline
\end{tabular}

$N_{\text {Kinder }}=995, N_{\text {Klassen }}=45$

$* * * p<0,001, * * p<0,01, * p<0,05$

${ }^{a}$ Für SDQ und Leistung wurde pro Klasse der Mittelwert (MW) über alle Schüler/innen berechnet

${ }^{b}$ Kohäsion_GI und_Dichte wurden je nach abhängiger Variable in der social- bzw. task-Variante genutzt 
In einem zweiten Analyseschritt wurde überprüft, für welche Individualmerkmale der Zusammenhang mit dem jeweiligen Indikator der Partizipation zwischen den Klassen (signifikant) variiert. Signifikante Varianzanteile ergeben sich für alle vier Partizipationsfacetten insbesondere für Geschlecht und SDQ (vgl. Tab. 4).

Die Ergebnisse der Zwei-Ebenen-Regressionsanalysen sind in Tab. 5 zusammengefasst.

Auf Individualebene ergeben sich im außerunterrichtlichen wie im unterrichtlichen Kontext sowohl für Akzeptanz als auch für Ablehnung signifikante Effekt des Migrationshintergrunds und der Verhaltensauffälligkeit. Im Vergleich zu ihren Mitschüler/innen derselben Klasse werden Kinder mit Migrationshintergrund und solche mit problematischem Verhalten (SDQ) seltener als Spiel- oder Arbeitspartner/innen akzeptiert und häufiger explizit abgelehnt. Ein Zusammenhang von Akzeptanz/Ablehnung mit dem Geschlecht ist nicht nachweisbar. Die Schulleistung erweist sich als prädiktiv für die Akzeptanz, nicht aber für die Ablehnung. Der Anteil der Varianz auf Ebene der Schüler/innen, der durch die Individualmerkmale erklärt wird, beträgt 37,7\% für die Akzeptanz und 41,5\% für die Ablehnung als Spielpartner/in sowie 54,0\% respektive 54,4\% für die Akzeptanz bzw. Ablehnung als Arbeitspartner/in.

Der Grad der Akzeptanz und der Ablehnung variiert deutlich zwischen den Klassen. Diese Variationen können je nach Partizipationsfacette durch die hier berücksichtigten Prädiktoren auf Gruppenebene in unterschiedlicher Weise erklärt werden. Die Klassenunterschiede in den Akzeptanzwerten in Spielsituationen können durch den Anteil an Kindern mit Migrationshintergrund und durch die Kohäsion in der Klasse erklärt werden. Je höher der Migrant/innenanteil und je kohäsiver die Klasse, desto höher fallen die Akzeptanzwerte aus. Sowohl das sozio- als auch das psychometrische Maß leisten hier einen eigenständigen Beitrag zur Vorhersage. Unterschiede zwischen den Klassen im Mädchenanteil, im durchschnittlichen Leistungsniveau oder im Maß an Verhaltensproblemen leisten hingegen keinen Beitrag zur Erklärung der Unterschiede in der mittleren klassenweisen Akzeptanz. Mit Blick auf die Ablehnung in Spielsituationen fällt die Befundlage etwas anders aus. Die Kohäsion hat hier einen geringeren Effekt, und nur das psychometrische Maß ist signifikant. In Bezug auf Kompositionseffekte zeigen sich lediglich Zusammenhänge mit dem klassenspezifischen Leistungsniveau und dem Anteil an Kindern mit Migrationshintergrund in der Klasse. In leistungsstarken Klassen und in solchen mit einem hohen Anteil an Kindern mit Migrationshintergrund werden Kinder insgesamt seltener explizit als Spielpartner/innen abgelehnt. Die Klassenunterschiede in der Akzeptanz in Arbeitssituationen können nicht durch Kompositionsmerkmale erklärt werden. Einzig die Kohäsion - erfasst über die Dichte - stellt einen signifikanten positiven Prädiktor für die mittlere Akzeptanz in den Klassen dar. Hinsichtlich der Ablehnung in Arbeitssituationen erweist sich das psychometrische Maß für die Kohäsion (Kohäsion_GI) als signifikant. In Klassen, in denen die Kinder einen guten Zusammenhalt im Unterricht wahrnehmen, ist die explizite Ablehnung von Mitschüler/innen als Arbeitspartner/innen geringer ausgeprägt. Zusammenhänge zeigen sich auch mit dem klassenspezifischen Leistungsniveau und dem Anteil an Kindern mit Migrationshintergrund in der Klasse. Je leistungsstärker die Klasse, desto seltener werden Kinder explizit als Arbeitspartner/innen abgelehnt, und auch mit einem höheren Anteil an 
Kindern mit Migrationshintergrund geht ein geringeres Ablehnungsniveau einher. Bedeutsame Cross-Level-Interaktionen ergeben sich nicht.

\section{Diskussion}

Erwartungsgemäß bestätigen die Ergebnisse, dass Kinder mit auffälligem Verhalten seltener als präferierte Spiel- und Arbeitspartner/in benannt und darüber hinaus öfter explizit abgelehnt werden. Die Schulleistung erweist sich zwar als negativer Prädiktor der Akzeptanz, zeigt aber keinen statistisch bedeutsamen Zusammenhang mit der Ablehnung auf. Dabei zeigt sich, entgegen unserer Erwartung, keine Differenzierung zwischen den beiden Kontexten (task und social). Die Ergebnisse bestätigen weiterhin, dass Klassenunterschiede in der Akzeptanz und der Ablehnung in Teilen durch Merkmale der Klassenkomposition erklärt werden können. Darüber hinaus erweist sich die Kohäsion als bedeutsamer Prädiktor. Wenngleich Kinder in kohäsiven Klassen insgesamt mehr Akzeptanz und weniger Ablehnung erfahren, profitieren Kinder mit schwachen Leistungen oder auffälligem Verhalten entgegen unserer Erwartung nicht stärker von hoher Kohäsion als ihre Mitschüler/innen.

Der SDQ-Wert als Maß für Verhaltensauffälligkeiten stellt für Akzeptanz und Ablehnung einen zentralen Prädiktor dar. Damit kann geschlossen werden, dass Kinder mit besonderem Unterstützungsbedarf im emotional-sozialen Bereich - wie auch bereits in anderen Studien gezeigt - eine besondere Risikogruppe darstellen (z. B. Krull et al. 2018). Das Leistungsmaß ist hingegen nur für die Akzeptanz prädiktiv, sodass abgeleitet werden kann, dass Kinder mit Lernschwierigkeiten weniger als Kinder mit auffälligem Verhalten von expliziter Ausgrenzung betroffen sind. Wenngleich nicht Fokus unserer Untersuchung, ist anzumerken, dass bei Kontrolle von Leistung und Verhaltensauffälligkeiten auch der Migrationshintergrund ein weiterer Risikofaktor für die Partizipation in Spiel- und Arbeitssituationen darstellt. Obwohl hierbei eine Konfundierung mit dem sozioökonomischen Status zu vermuten ist, kann festgehalten werden, dass nach unseren Befunden Kinder mit Verhaltensauffälligkeiten und Kinder mit Migrationshintergrund besonders in den Blick zu nehmen sind, damit Inklusion im Sinne einer gelingenden Partizipation aller Schüler/innen gelingen kann.

Die Schulklassen unterscheiden sich selbst nach Kontrolle der Individualmerkmale noch deutlich in ihrem mittleren Wert der Akzeptanz bzw. Ablehnung. Gerade in den außerunterrichtlichen Situationen, die zumeist nicht unter Leitung der Lehrkraft stehen, liegen hohe Akzeptanz- und geringe Ablehnungswerte eher in hoch kohäsiven Klassengemeinschaften vor. In Klassen, in denen ein guter Zusammenhalt herrscht, gelingt es den Kindern besser, auch in Situationen ohne Steuerung durch eine Lehrkraft Ausgrenzung zu vermeiden und soziale Akzeptanz herzustellen. Dies kann möglicherweise darauf zurückgeführt werden, dass bei den Kindern entsprechende Normen und Werte, die im Unterricht gelebt werden, etabliert sind.

Damit rückt die Rolle der Lehrkraft in den Fokus. In bisherigen Studien hat sich beispielsweise das Lehrkraftfeedback als wichtiges Merkmal erwiesen, über das die Partizipation von Kindern mit besonderem Unterstützungsbedarf gestärkt werden kann. Gemäß der sozialen Referenzierungstheorie (Webster und Foschi 1992) ori- 
entieren sich Schüler/innen in der Akzeptanz bzw. Ablehnung eines anderen Kindes an der Art des Lehrkraftfeedbacks zu diesem Kind (Huber 2011; Huber et al. 2015, 2018; Schwab et al. 2015). Mit einer gezielten Einflussnahme auf den Zusammenhalt der Klasse, beispielsweise über Teambuildingmaßnahmen, erhält die Lehrkraft möglicherweise ein weiteres Instrument, soziale Partizipation positiv zu beeinflussen. Der Fokus auf die Klasse als Ganzes erscheint mit Blick auf den inklusiven Grundgedanken besonders passend, da das (negative) Herausheben Einzelner und Stigmatisierung auf diese Weise vermieden wird.

Gleichzeitig ist jedoch auch im Blick zu halten, dass mit der Kohäsion zwar eine Stellschraube gefunden wurde, die generell Einfluss auf die Akzeptanz und Ablehnung nimmt, aber nicht speziell positiven Einfluss auf die Akzeptanz und Ablehnung von Kindern mit besonderem Unterstützungsbedarf hat. Insbesondere für Kinder mit externalisierenden Störungsbildern sind sicherlich Multikomponentenansätze (Garrote et al. 2017) notwendig, die beispielweise Sozialkompetenztrainings beinhalten, um die Akzeptanz dieser Kinder zu erhöhen und insbesondere ihre Ablehnung zu verringern. Entgegen unserer Erwartung und der Befunde von Schürer (2019) konnten wir für die Akzeptanz und die Ablehnung keine systematischen Unterschiede zwischen den Kontexten der Partizipation (task vs. social) finden. Dies ist möglichweise durch die unterschiedlichen Operationalisierungen des Konstrukts der sozialen Partizipation zu erklären. Zu fordern ist eine tiefere, theoretische Auseinandersetzung mit diesem sehr facettenreichen Konstrukt. Wann kann von gelungener Partizipation gesprochen werden? Eine hohe Akzeptanz und geringe Ablehnung sind sicherlich grundlegende Indikatoren gelungener Partizipation. Spricht aber die Quantität erhaltener Wahlen auch für die Qualität der dahinterliegenden Beziehungen? Oder sind ein oder zwei reziproke Beziehungen ausreichend, um das Bedürfnis nach Zugehörigkeit zu befriedigen? Wie stehen Akzeptanz und Ablehnung zueinander? Welche Bedeutung haben verwandte Konstrukte, wie z. B. Wertschätzung? Eine elaborierte Auseinandersetzung und theoretische Rahmung fehlen bislang. Hierzu gehört auch, die verschiedenen Möglichkeiten der Operationalisierung sozialer Partizipation mit dieser theoretischen Rahmung zu verknüpfen. In unseren Analysen wurde mit der Akzeptanz bzw. Ablehnung nur ein Indikator der Partizipation in den Blick genommen. Die Befunde bestätigen, dass Kinder weniger akzeptiert und häufiger abgelehnt werden, je mehr Unterstützungsbedarf sie haben. Damit ist jedoch nicht gesagt, dass diese Kinder nicht auch in Freundschaften oder kohäsive Subgruppen eingebunden sind, sodass nicht zwangsläufig von einer Isolation dieser Kinder gesprochen werden kann. Hier sind weitere Analysen mit den Daten erforderlich, um die Netzwerkstrukturen der Klasse detaillierter zu untersuchen.

Gleiches ist auch für das Konstrukt der Kohäsion zu fordern, das bislang nur in wenigen schulbezogene Studien in elaborierter Form Eingang gefunden hat (Begert 2019; Schürer 2019). Das hier genutzte Maß der Dichte ist zwar ein gängiges Kohäsionsmaß in der sozialen Netzwerkanalyse, allerdings gibt es nur Auskunft über die Anzahl an Relationen und nicht zu ihrer Verteilung oder zu Distanzen. Weitere Indizes (z. B. Clustering-Indizes, Prell 2012), die in einem theoretischen Modell der Kohäsion im schulischen Kontext verankert sind, könnten differenziertere Auskunft geben. 
Es ist festzuhalten, dass wir im Rahmen unserer Studie, wie es auch in vielen anderen Studien der Fall ist, mit der Problematik konfrontiert sind, keine direkten Aussagen zur sozialen Partizipation von Kindern mit SPF tätigen zu können. Insbesondere im Grundschulbereich besteht für empirische Studien häufig gar nicht die Möglichkeit der Nutzung eines diagnostizierten SPFs, da ein Verfahren zur Feststellung eines solchen im FSP LE beispielsweise in vielen Bundesländern erst nach dem dritten Jahr in der Schuleingangsphase eröffnet wird. Ein Feststellungsverfahren für den FSP ESE wird teilweise nur dann eröffnet, wenn mit diesem eine Selbst- oder Fremdgefährdung einhergeht (z.B. Ministerium für Schule und Weiterbildung des Landes Nordrhein-Westfalen 2016, Schulgesetz $§ 19$ (7)). Daher ist einschränkend festzuhalten, dass wir allein mit der Lehrkraftversion des SDQ und standardisierten Leistungstests das komplexe Konstrukt des SPFs nicht abbilden können. Bewusst haben wir uns jedoch für ein kontinuierliches Maß entschieden, um zum einen entsprechend dem Inklusionsgedanken keine Kategorisierung vorzunehmen und zum anderen Aussagen zum Zusammenhang von Leistung und Verhaltensauffälligkeit sowie der Partizipation treffen zu können. Die hier genutzte Herangehensweise, Leistung und Verhaltensauffälligkeiten über metrische Variablen zu codieren und mit den Partizipationsmaßen in Beziehung zu setzen, setzt jedoch stillschweigend einen linearen - also über den ganzen Wertebereich gleichmäßigen - Zusammenhang voraus. Denkbar ist jedoch, dass ein bestimmter Schwellenwert überschritten werden muss, bevor Leistungsdefizite und problematische Verhaltensweisen einen Effekt auf die Partizipation haben. Weitere Analysen mit verschiedenen ,cut-offpoints“ zur Bildung kategorialer Variablen, könnten eine solche Annahme überprüfen.

Trotz dieser Limitationen unserer Studie bestätigen die Befunde dennoch insgesamt, dass Kinder mit schwachen Schulleistungen und emotional-sozial auffälligem Verhalten zu einer Risikogruppe bzgl. sozialer Partizipation gehören. Unsere Ergebnisse zeigen jedoch auch Möglichkeiten auf, wie Lehrkräfte gezielt Einfluss nehmen können, um soziale Partizipation in den Klassen zu verbessern. Mit der Kohäsion der Klasse ist eine Stellschraube gefunden, die Einfluss auf die Akzeptanz und Ablehnung nimmt, ohne das individuelle Kind $\mathrm{zu}$ fokussieren und somit ggf. zu stigmatisieren. Auch Kompositionsmerkmale sind mögliche Prädiktoren der Partizipation. So scheint ein hoher Migrationsanteil eher förderlich für hohe Akzeptanz bzw. geringe Ablehnung zu sein. In weiteren Studien sollten daher vermehrt die Klassenmerkmale in den Blick genommen werden.

Funding Open Access funding enabled and organized by Projekt DEAL.

Open Access Dieser Artikel wird unter der Creative Commons Namensnennung 4.0 International Lizenz veröffentlicht, welche die Nutzung, Vervielfältigung, Bearbeitung, Verbreitung und Wiedergabe in jeglichem Medium und Format erlaubt, sofern Sie den/die ursprünglichen Autor(en) und die Quelle ordnungsgemäß nennen, einen Link zur Creative Commons Lizenz beifügen und angeben, ob Änderungen vorgenommen wurden.

Die in diesem Artikel enthaltenen Bilder und sonstiges Drittmaterial unterliegen ebenfalls der genannten Creative Commons Lizenz, sofern sich aus der Abbildungslegende nichts anderes ergibt. Sofern das betreffende Material nicht unter der genannten Creative Commons Lizenz steht und die betreffende Handlung nicht nach gesetzlichen Vorschriften erlaubt ist, ist für die oben aufgeführten Weiterverwendungen des Materials die Einwilligung des jeweiligen Rechteinhabers einzuholen. 
Weitere Details zur Lizenz entnehmen Sie bitte der Lizenzinformation auf http://creativecommons.org/ licenses/by/4.0/deed.de.

\section{Literatur}

Avramidis, E. (2013). Self-concept, social position and social participation of pupils with SEN in mainstream primary schools. Research Papers in Education, 28(4), 421-442. https://doi.org/10.1080/ 02671522.2012 .673006$.

Baumeister, R.F., \& Leary, M.R. (1995). The need to belong: desire for interpersonal attachments as a fundamental human motivation. Psychological Bulletin, 117(3), 497-529. https://doi.org/10.1037/ 0033-2909.117.3.497.

Baumeister, R.F., DeWall, C. N., Ciarocco, N. J., \& Twenge, J.M. (2005). Social exclusion impairs selfregulation. Journal of Personality and Social Psychology, 88(4), 589-604. https://doi.org/10.1037/ 0022-3514.88.4.589.

Begert, T. (2019). Klassenzusammenhalt und schulisches Problemverhalten: Eine netzwerkanalytische Untersuchung auf der Sekundarstufe I. Berlin Heidelberg: Springer. https://doi.org/10.1007/978-3-65824050-9.

Bless, G. (2000). Lernbehinderungen. In J. Borchert (Hrsg.), Handbuch der Sonderpädagogischen Psychologie (S. 440-453). Hogrefe.

Bless, G., \& Mohr, K. (2007). Die Effekte von Sonderunterricht und gemeinsamem Unterricht auf die Entwicklung von Kindern mit Lernbehinderungen. In J. Walter \& F. B. Wember (Hrsg.), Sonderpädagogik des Lernens (S. 375-383). Hogrefe.

Borgatti, S.P., Everett, M. G., \& Johnson, J.C. (2018). Analyzing social networks. SAGE.

Cambra, C., \& Silvestre, N. (2003). Students with special educational needs in the inclusive classroom: social integration and self-concept. European Journal of Special Needs Education, 18(2), 197-208. https://doi.org/10.1080/0885625032000078989.

Carron, A. V., Brawley, L.R., \& Widmeyer, W.N. (1998). The measurement of cohesiveness in sport groups. In J. L. Duda (Hrsg.), Advances in sport and exercise psychology measurement (S. 213-226). Fitness Information Technology.

Cook, B. G., \& Semmel, M.I. (1999). Peer acceptance of included students with disabilities as a function of severity of disability and classroom composition. Journal of Special Education, 33(1), 50-61.

Dumont, H., Neumann, M., Maaz, K., \& Trautwein, U. (2013). Die Zusammensetzung der Schülerschaft als Einflussfaktor für Schulleistungen. Internationale und nationale Befunde. Internationale und nationale Befunde. Psychologie in Erziehung und Unterricht, 60(3), 163-183.

Frederickson, N., \& Furnham, A.F. (2001). The long-term stability of sociometric status classification: a longitudinal study of included pupils who have moderate learning difficulties and their mainstream peers. Journal of Child Psychology and Psychiatry, 42(5), 581-592.

Frederickson, N., \& Graham, B. (1999). Social skills and emotional intelligence. NFER-Nelson.

Garrote, A., Sermier Dessemontet, R., \& Opitz, M.E. (2017). Facilitating the social participation of pupils with special educational needs in mainstream schools: a review of school-based interventions. Educational Research Review, 20, 12-23. https://doi.org/10.1016/j.edurev.2016.11.001.

Goodman, R. (1997). The strengths and difficulties questionnaire: a research note. Journal of Child Psychology and Psychiatry, 28(5), 581-586. https://doi.org/10.1111/j.1469-7610.1997.tb01545.x.

Grütter, J., Meyer, B., \& Glenz, A. (2015). Sozialer Ausschluss in Integrationsklassen: Ansichtssache? Psychologie in Erziehung und Unterricht, 62(1), 65-82. https://doi.org/10.2378/peu2015.art05d.

Hannover, B., \& Zander, L. (2016). Die Bedeutung der Peers für die individuelle schulische Entwicklung. In J. Möller, M. Köller \& T. Riecke-Baulecke (Hrsg.), Basiswissen Lehrerbildung: Schule und Unterricht Lehren und Lernen (S. 91-105). Klett, Kallmeyer.

Hawkley, L. C., \& Cacioppo, J. T. (2010). Loneliness matters: A theoretical and empirical review of consequences and mechanisms. Annals of Behavioral Medicine, 40(2), 218-227. https://doi.org/10.1007/ s12160-010-9210-8.

Henke, T., Bogda, K., Lambrecht, J., Bosse, S., Koch, H., Maaz, K., \& Spörer, N. (2017). Will you be my friend? A multilevel network analysis of friendships of students with and without special educational needs backgrounds in inclusive classrooms. Zeitschrift für Erziehungswissenschaft, 20(3), 449-474. https://doi.org/10.1007/s11618-017-0767-x.

Huber, C. (2011). Lehrerfeedback und soziale Integration: Wie soziale Referenzierungsprozesse die soziale Integration in der Schule beeinflussen könnten. Empirische Sonderpädagogik, 3(1), 20-36. 
Huber, C., \& Wilbert, J. (2012). Soziale Ausgrenzung von Schülern mit sonderpädagogischem Förderbedarf und niedrigen Schulleistungen im gemeinsamen Unterricht. Empirische Sonderpädagogik, 4(2), $147-165$.

Huber, C., Gebhardt, M., \& Schwab, S. (2015). Lehrkraftfeedback oder Spaß beim Spiel? Eine Experimentalstudie zum Einfluss von Lehrkraftfeedback auf die soziale Akzeptanz bei Grundschulkindern. Psychologie in Erziehung und Unterricht, 62(1), 51-64. https://doi.org/10.2378/peu2015.art04d.

Huber, C., Gerullis, A., Gebhardt, M., \& Schwab, S. (2018). The impact of social referencing on social acceptance of children with disabilities and migrant background: An experimental study in primary school settings. European Journal of Special Needs Education, 33(2), 269-285. https://doi.org/10. 1080/08856257.2018.1424778.

Jansen, D. (2006). Einführung in die Netzwerkanalyse: Grundlagen, Methoden, Forschungsbeispiele. VS.

Kavale, K. A., \& Forness, S. R. (1996). Social skill deficits and learning disabilities: a meta-analysis. Journal of Learning Disabilities, 29(3), 226-237. https://doi.org/10.1177/002221949602900301.

Koster, M., Nakken, H., Pijl, S.J., \& van Houten, E. J. (2009). Being part of the peer group: a literature study focusing on the social dimension of inclusion in education. International Journal of Inclusive Education, 13(2), 117-140. https://doi.org/10.1080/13603110701284680.

Krajewski, W., Küspert, P., \& Schneider, W. (2002a). Deutscher Mathematiktest für erste Klassen (DEMAT $1+)$. Hogrefe.

Krajewski, W., Liehm, S., \& Schneider, W. (2002b). Deutscher Mathematiktest für zweite Klassen (DEMAT 2+). : Hogrefe.

Krawinkel, S., Südkamp, A., Lange, S., \& Tröster, H. (2017). Soziale Partizipation in inklusiven Grundschulklassen: Bedeutung von Klassen und Lehrkraftmerkmalen. Empirische Sonderpädagogik, 3, 277-295.

Krull, J., Wilbert, J., \& Hennemann, T. (2014). Soziale Ausgrenzung von Erstklässlerinnen und Erstklässlern mit sonderpädagogischem Förderbedarf im Gemeinsamen Unterricht. Empirische Sonderpädagogik, 6(1), 59-75.

Krull, J., Wilbert, J., \& Hennemann, T. (2018). Does social exclusion by classmates lead to behaviour problems and learning difficulties or vice versa? A cross-lagged panel analysis. European Journal of Special Needs Education, 33(2), 235-253. https://doi.org/10.1080/08856257.2018.1424780.

Kulawiak, P.R., \& Wilbert, J. (2015). Methoden zur Analyse der sozialen Integration von Schulkindern mit sonderpädagogischem Förderbedarf im gemeinsamen Unterricht. Empirische Sonderpädagogik, 7(3), 241-257.

Lenhard, W., Lenhard, A., \& Schneider, W. (2017). ELFE II. Ein Leseverständnistest für Erst- bis Siebtklässler - Version II. Hogrefe.

Mand, J. (2007). Social position of special needs pupils in the classroom: a comparison between German special schools for pupils with learning difficulties and integrated primary school classes. European Journal of Special Needs Education, 22(1), 7-14. https://doi.org/10.1080/08856250601082182.

Ministerium für Schule und Weiterbildung des Landes Nordrhein-Westfalen (2016). Schulgesetz für das Land Nordrhein-Westfalen. https://bass.schul-welt.de/6043.htm. Zugegriffen: 05.01.2022.

Monchy, M.D., Pijl, S. J., \& Zandberg, T. (2004). Discrepancies in judging social inclusion and bullying of pupils with behaviour problems. European Journal of Special Needs Education, 19(3), 317-330. https://doi.org/10.1080/0885625042000262488.

Nepi, L. D., Facondini, R., Nucci, F., \& Peru, A. (2013). Evidence from full-inclusion model: The social position and sense of belonging of students with special educational needs and their peers in Italian primary school. European Journal of Special Needs Education, 28(3), 319-332. https://doi.org/10. 1080/08856257.2013.777530.

Nepi, L. D., Fioravanti, J., Nannini, P., \& Peru, A. (2015). Social acceptance and the choosing of favourite classmates: a comparison between students with special educational needs and typically developing students in a context of full inclusion. British Journal of Special Education, 42(3), 319-337. https:// doi.org/10.1111/1467-8578.12096.

Nowicki, E. A. (2003). A meta-analysis of the social competence of children with learning disabilities compared to classmates of low and average to high achievement. Learning Disabilities Quarterly, 26(3), 171-188. https://doi.org/10.2307/1593650.

Pijl, S.J., Frostad, P., \& Flem, A. (2008). The social position of pupils with special needs in regular schools. Scandinavian Journal of Educational Research, 52(4), 387-405. https://doi.org/10.1080/ 00313830802184558.

Prell, C. (2012). Social network analysis: history, theory \& methodology. SAGE.

Raudenbush, S.W., Bryk, A.S., \& Congdon, R. (2009). HLM for windows (6.0. 8)[software. Scientific Software International. 
Rauer, W., \& Schuck, K. D. (2003). Fragebogen zur Erfassung emotionaler und sozialer Schulerfahrungen von Grundschulkindern dritter und vierter Klassen: FEESS 3-4; Manual. Beltz.

Scharenberg, K., Rollett, W., \& Bos, W. (2019). Do differences in classroom composition provide unequal opportunities for academic learning and social participation of SEN students in inclusive classes in primary school? School Effectiveness and School Improvement, 30(3), 309-327. https://doi.org/10. 1080/09243453.2019.1590423.

Schürer, S. (2020). Soziale Partizipation von Kindern mit sonderpädagogischem Förderbedarf in den Bereichen Lernen und emotional-soziale Entwicklung in der allgemeinen Grundschule - Ein Literaturreview. Empirische Sonderpädagogik, 12(4), 295-319.

Schürer, S., van Ophuysen, S., \& Behrmann, L. (2020). Cohesion in classes of primary and secondary school-presentation of an instrument and assessment of measurement invariance. Journal of Psychoeducational Assessment. https://doi.org/10.1177/0734282920966825.

Schürer, S. (2019). Der Einfluss von Gruppenkohäsion auf die soziale Partizipation individueller Schülerinnen und Schüler. Eine Interventionsstudie an inklusiv unterrichtenden Grundschulen [Dissertationsschrift]. Westfälische Wilhelms-Universität Münster. https://d-nb.info/1193126185/34. Zugegriffen: 05.01.2022.

Schwab, S., Huber, C., \& Gebhardt, M. (2015). Social acceptance of students with down syndrome and students without disability. Educational Psychology, 36(8), 1501-1515. https://doi.org/10.1080/ 01443410.2015 .1059924$.

Spilles, M., Hagen, T., \& Hennemann, T. (2019). Wirkungen einer tutoriellen Leseflüssigkeitsförderung auf die soziale Integration von Kindern mit externalisierenden Verhaltensproblemen sowie auf die Lesegeschwindigkeit von Tutoren und Tutanden. Vierteljahresschrift für Heilpädagogik und ihre Nachbargebiete, 88(1), 44-57.

Van Ewijk, R., \& Sleegers, P. (2010). Peer ethnicity and achievement: a meta-analysis into the compositional effect. School Effectiveness and School Improvement, 21(3), 237-265. https://doi.org/10.1080/ 09243451003612671.

Webster, M., \& Foschi, M. (1992). Social referencing and theories of status and social interaction. In S. Feinman (Hrsg.), Social referencing and the social construction of reality in infancy (S. 269-296). Plenum Press.

Wentzel, K. R., Baker, S., \& Russell, S. (2014). Peer relationships and positive adjustment at school. In M. J. Furlong, R. Gilman \& E.S. Huebner (Hrsg.), Educational psychology handbook series. Handbook of Positive Psychology in Schools. (S. 260-277). Routledge.

Wentzel, K. R., Russell, S., \& Baker, S. (2016). Emotional support and expectations from parents, teachers, and peers predict adolescent competence at school. Journal of Educational Psychology, 108(2), 242-255. https://doi.org/10.1037/edu0000049.

Williams, K. D. (2007). Ostracism. Annual Review of Psychology, 58, 425-452. https://doi.org/10.1146/ annurev.psych.58.110405.085641.

Zurbriggen, C., \& Venetz, M. (2016). Soziale Partizipation und aktuelles Erleben im gemeinsamen Unterricht. Empirische Pädagogik, 30(1), 98-112. 